\title{
A WEIGHTED INEQUALITY FOR THE KAKEYA MAXIMAL OPERATOR
}

\author{
A. M. VARGAS
}

(Communicated by J. Marshall Ash)

\begin{abstract}
Let $\mathscr{K}_{\delta}$ be the Kakeya Maximal Operator defined as the supremum of averages over parallelepipeds of eccentricity $\delta$. We show that $\mathscr{K}_{\delta}$ satisfies $\left\|\mathscr{K}_{\delta} f\right\|_{L^{p}(\omega)} \leq C_{n, p}(1 / \delta)^{n / p-1}(\log (1 / \delta))^{\alpha_{n}}\|f\|_{L^{p}\left(\mathscr{R}_{\delta} \omega\right)}$ for all $p \leq(n+1) / 2$ with some constants $C_{n, p}, \alpha_{n}$, independent of $f$ and the weight $\omega$.
\end{abstract}

For $0<\delta<1$ and $f \in L_{\mathrm{loc}}^{1}\left(\mathbf{R}^{n}\right)$, we define the Kakeya Maximal Function of eccentricity $\delta$

$$
\mathscr{K}_{\delta} f(x)=\sup \frac{1}{\left|R_{x}\right|} \int_{R_{x}}|f(y)| d y,
$$

where the 'sup' is taken over all rectangular parallelepipeds in $\mathbf{R}^{n}$ homothetic to $[0, \delta]^{n-1} \times[0,1]$ of arbitrary direction and containing $x$.

It is conjectured that $\mathscr{K}_{\delta}$ is bounded on $L^{n}\left(\mathbf{R}^{n}\right)$ with a constant given by

$$
C_{n}\left(\log \frac{1}{\delta}\right)^{\alpha_{n}}
$$

for some $C_{n}, \alpha_{n}>0$.

If the conjecture were true,we would obtain, by interpolation, the following $L^{p}$ estimates:

$$
\left\|\mathscr{K}_{\delta} f\right\|_{L^{p}\left(\mathbf{R}^{n}\right)} \leq \begin{cases}C_{n}\left(\log \frac{1}{\delta}\right)^{\alpha_{n}}\|f\|_{L^{p}\left(\mathbf{R}^{n}\right)} & \text { for } p \geq n, \\ C_{n, p}\left(\frac{1}{\delta}\right)^{n / p-1}\left(\log \frac{1}{\delta}\right)^{\alpha_{n}}\|f\|_{L^{p}\left(\mathbf{R}^{n}\right)} & \text { for } 1<p<n .\end{cases}
$$

To see it, note that the Kakeya operator is bounded on $L^{\infty}\left(\mathbf{R}^{n}\right)$ with constant 1 and is of weak-type 1 with constant $C_{n}\left(\frac{1}{\delta}\right)^{n-1}$. (The latter follows from the classical result for the Hardy-Littlewood maximal operator.)

For $n=2$ the conjecture is known to be true (see [C]). More recently, the following double-weight $L^{2}$ estimate has been proved

$$
\left\|\mathscr{K}_{\delta} f\right\|_{L^{2}(\omega)} \leq C\left(\log \frac{1}{\delta}\right)^{\alpha}\|f\|_{L^{2}\left(\mathscr{H}_{\delta} \omega\right)}
$$

for all weights $\omega$ in $\mathbf{R}^{2}$ (see [MS]).

Received by the editors January 6, 1992 and, in revised form, July 8, 1992.

1991 Mathematics Subject Classification. Primary 42B25.

Key words and phrases. Maximal functions, weighted inequalities. 
In higher dimensions the question is still open. Christ, Duoandikoetxea, and Rubio de Francia [CDR] established (2) for $p \leq \frac{n+1}{2}$. Recently, Bourgain [B] proved that there is an exponent $p_{n}>\frac{n+1}{2}$ so that (2) holds for $p=p_{n}$.

In this note we establish the following weighted version of the result in [CDR]

Theorem 1. For $n \geq 3$ and $1<p \leq \frac{n+1}{2}$ there are constants $C_{n, p}, \alpha_{n}>0$ such that, for every $1>\delta>0$ and every positive weight $\omega$, we have

$$
\left\|\mathscr{K}_{\delta} f\right\|_{L^{p}(\omega)} \leq C_{n, p}\left(\frac{1}{\delta}\right)^{n / p-1}\left(\log \frac{1}{\delta}\right)^{\alpha_{n}}\|f\|_{L^{p}\left(\mathscr{K}_{\delta} \omega\right)} .
$$

Let us consider another maximal function

$$
\mathscr{M}_{\delta} f(x)=\sup \frac{1}{\left|R_{x}\right|} \int_{R_{x}}|f(y)| d y,
$$

where the 'sup' is taken over rectangular parallelepipeds in $\mathbf{R}^{n}$, containing $x$, regardless of orientation, which have one side of length 1 and $n-1$ sides, each of length $\delta$.

By interpolation and a 'sieve' argument, which will be discussed later, Theorem 1 is a consequence of the following restricted weak-type estimate:

Theorem 2. There is a constant $C_{n}>0$ such that

$$
\omega\left(\left\{x: \mathscr{M}_{\delta}\left(\chi_{A}\right)(x)>\sigma\right\}\right) \leq C_{n} \sigma^{-(n+1) / 2} \delta^{-(n-1) / 2} \mathscr{K}_{\delta} \omega(A),
$$

for all $A \subset B(0,1), \sigma>0,1>\delta>0$.

Proof of Theorem 2. For every $y \in\left\{x: \mathscr{M}_{\delta}\left(\chi_{A}\right)(x)>\sigma\right\}$ we select a parallelepiped, $R_{y}$, as in the definition of the maximal function and such that $\left|R_{y} \cap A\right|>\sigma\left|R_{y}\right|$. Following Bourgain [B], for $x \in A$ we define the 'bush' centered at $x$ as $\mathscr{B}_{x}=\bigcup_{x \in R_{y}} R_{y}$. We want to show that there is a point $x_{o} \in A$ such that

$$
\omega\left(\mathscr{B}_{x_{o}}\right) \geq c_{n} \sigma \delta^{n-1} \frac{\omega\left(\left\{x: \mathscr{M}_{\delta}\left(\chi_{A}\right)(x)>\sigma\right\}\right)}{|A|} .
$$

To see this, observe that

$$
\begin{aligned}
\int_{A} \omega\left(\mathscr{B}_{x}\right) d x & =\int_{A} \int_{\mathbf{R}^{n}} \chi_{\mathscr{B}_{x}}(y) \omega(y) d y d x \\
& \geq \int_{\left\{x: \mathscr{M}_{\delta}\left(\chi_{A}\right)(x)>\sigma\right\}} \omega(y) \int_{A} \chi_{\mathscr{B}_{x}}(y) d x d y .
\end{aligned}
$$

Now, note that, if $\mathscr{M}_{\delta}\left(\chi_{A}\right)(y)>\sigma$ and $x \in R_{y}$, then $y \in \mathscr{B}_{x}$, so that $\chi_{\mathscr{B}_{x}}(y) \geq$ $\chi_{R_{y}}(x)$. Hence,

$$
\begin{aligned}
\int_{A} \omega\left(\mathscr{B}_{x}\right) d x & \geq \int_{\left\{x: \mathscr{M}_{\delta}\left(\chi_{A}\right)(x)>\sigma\right\}} \omega(y) \int_{A} \chi_{R_{y}}(x) d x d y \\
& \geq \int_{\left\{x: \mathscr{M}_{\delta}\left(\chi_{A}\right)(x)>\sigma\right\}} \omega(y)\left|R_{y} \cap A\right| d y \\
& \geq \int_{\left\{x: \mathscr{K}_{\delta}\left(\chi_{A}\right)(x)>\sigma\right\}} \omega(y) c_{n} \sigma \delta^{n-1} d y \\
& =c_{n} \sigma \delta^{n-1} \omega\left(\left\{x: \mathscr{M}_{\delta}\left(\chi_{A}\right)(x)>\sigma\right\}\right),
\end{aligned}
$$

and we obtain what we wanted. 
On the other hand, suppose we had a set $\mathscr{E} \subset\left\{x: \mathscr{M}_{\delta}\left(\chi_{A}\right)(x)>\sigma\right\}$ and a point $y_{o}$ verifying

$$
\begin{aligned}
& \text { (a) } y_{o} \in \bigcap_{y \in \mathscr{E}} R_{y}, \\
& \text { (b) } \operatorname{ang}\left(R_{y}, R_{z}\right) \geq 10 \delta / \sigma \quad \text { if } y, z \in \mathscr{E}, y \neq z .
\end{aligned}
$$

(By $\operatorname{ang}\left(R_{y}, R_{z}\right)$ we denote the angle beetwen the main axes of $R_{y}$ and $R_{z}$.)

Then, we could define $\mathscr{B}=\bigcup_{y \in \mathscr{E}} 3 R_{y}$, a star-like set with respect to $y_{o}$, which would satisfy

$$
\begin{aligned}
\omega(\mathscr{B}) & \leq \sum_{y \in \mathscr{E}} \omega\left(3 R_{y}\right)=\sum\left(\frac{1}{\left|3 R_{y}\right|} \int_{3 R_{y}} \omega\right) 3^{n}\left|R_{y}\right| \\
& \leq c_{n} \sum\left|R_{y}\right|\left(\inf _{R_{y}} \mathscr{K}_{\delta} \omega\right) .
\end{aligned}
$$

Recall $\left|R_{y} \cap A\right|>\sigma\left|R_{y}\right|$. Moreover, $\left|R_{y} \cap A \cap B\left(y_{o}, \frac{\sigma}{3}\right)\right| \leq\left|R_{y} \cap B\left(y_{o}, \frac{\sigma}{3}\right)\right| \leq$ $\frac{2 \sigma}{3}\left|R_{y}\right|$. ( $R_{y} \cap B\left(y_{o}, \frac{\sigma}{3}\right)$ is contained in a parallelepiped with one side of length $\frac{2 \sigma}{3}$ and $n-1$ sides of length $\delta$.) Therefore, $\left|R_{y} \cap A \backslash B\left(y_{o}, \frac{\sigma}{3}\right)\right|>\frac{\sigma}{3}\left|R_{y}\right|$, and then

$$
\begin{aligned}
\omega(\mathscr{B}) & \leq c_{n} \sum \frac{\left|R_{y} \cap A \backslash B\left(y_{o}, \sigma / 3\right)\right|}{\sigma}\left(\inf _{R_{y}} \mathscr{K}_{\delta} \omega\right) \\
& \leq \sum \frac{c_{n}}{\sigma} \mathscr{K}_{\delta} \omega\left(R_{y} \cap A \backslash B\left(y_{o}, \frac{\sigma}{3}\right)\right) \leq \frac{c_{n}}{\sigma} \mathscr{K}_{\delta} \omega(\mathscr{B} \cap A) .
\end{aligned}
$$

(The last inequality follows from the fact that the sets $\left\{R_{y} \cap A \backslash B\left(y_{o}, \frac{\sigma}{3}\right)\right\}_{y \in \mathscr{E}}$ are mutually disjoint.)

Let us define, for the point $x_{o}$ whose existence was proved above, the set

$$
\mathscr{E}_{x_{o}}=\left\{y: x_{o} \in R_{y}\right\}
$$

Now, a moment's thought will convince us that we can choose a subset $\mathscr{E} \subset \mathscr{E}_{x_{o}}$ satisfying (a), with respect to $x_{o}$, and (b), such that

$$
\omega\left(\bigcup_{y \in \mathscr{E}} 3 R_{y}\right) \geq c_{n} \omega\left(\mathscr{B}_{x_{o}}\right) \sigma^{n-1}
$$

Then, from the construction of $\mathscr{B}_{x_{o}}$, the definition of $\mathscr{E}$, and the above computation on the $\omega$-measure of $\mathscr{B}=\bigcup\left\{3 R_{y}: y \in \mathscr{E}\right\}$, we have

$$
\begin{aligned}
\frac{c_{n} \sigma \delta^{n-1} \omega\left(\left\{x: \mathscr{M}_{\delta}\left(\chi_{A}\right)(x)>\sigma\right\}\right)}{|A|} & \leq \omega\left(\mathscr{B}_{x_{o}}\right) \leq c_{n} \sigma^{1-n} \omega\left(\bigcup_{y \in \mathscr{E}} 3 R_{y}\right) \\
& \leq c_{n} \sigma^{-n} \mathscr{K}_{\delta} \omega(A \cap \mathscr{B}) \leq c_{n} \sigma^{-n} \mathscr{K}_{\delta} \omega(A) .
\end{aligned}
$$


Finally, if $x \in B(0,2)$, then $\mathscr{K}_{\delta} \omega(x) \geq c_{n} \omega(B(0,2))$. We deduce

$$
\mathscr{K}_{\delta} \omega(A) \geq c_{n} \omega(B(0,2))|A|,
$$

and, therefore,

$$
\begin{aligned}
& \frac{c_{n} \sigma \delta^{n-1} \omega\left(\left\{x: \mathscr{M}_{\delta}\left(\chi_{A}\right)(x)>\sigma\right\}\right)^{2}}{\mathscr{K}_{\delta} \omega(A)} \\
& \quad \leq \frac{c_{n} \sigma \delta^{n-1} \omega\left(\left\{x: \mathscr{M}_{\delta}\left(\chi_{A}\right)(x)>\sigma\right\}\right) \omega(B(0,2))}{\mathscr{K}_{\delta} \omega(A)} \\
& \quad \leq \frac{c_{n} \sigma \delta^{n-1} \omega\left(\left\{x: \mathscr{M}_{\delta}\left(\chi_{A}\right)(x)>\sigma\right\}\right)}{|A|} \leq c_{n} \sigma^{-n} \mathscr{K}_{\delta} \omega(A) .
\end{aligned}
$$

This finishes with the proof of Theorem 2.

Proof of Theorem 1. Estimate (4) follows from Theorem 2, by standard arguments. First, we prove a restricted weak-type inequality (analogous to (5)) for $\mathscr{K}_{\delta}$ :

$$
\omega\left(\left\{x: \mathscr{K}_{\delta}\left(\chi_{A}\right)(x)>\sigma\right\}\right) \leq C_{n} \sigma^{-(n+1) / 2} \delta^{-(n-1) / 2}\left(\log \frac{1}{\delta}\right)^{\beta_{n}} \mathscr{K}_{\delta} \omega(A) .
$$

In order to do that, we select some suitable families of parallelepipeds from the level set $\left\{\mathscr{K}_{\delta}\left(\chi_{A}\right)>\sigma\right\}$ and decompose $A$ into disjoint subsets associated to the families. Each parallelepiped of a family belongs to one of, essentially, $\log \frac{1}{\delta}$ level sets associated to $\mathscr{M}_{\delta}$ and the corresponding subset. (This 'sieve' argument can be seen in [C] and [MS].)

Last of all, we use a three-point interpolation lemma as in [C] or, more directly, as in Proposition 5 in [CHS]. This will give us the strong type estimate (4).

Remark. Define

$$
\mathscr{M}_{\delta}^{6} f(x)=\sup \frac{1}{\left|R_{x}\right|} \int_{R_{x}}|f(y)| d y,
$$

where the 'sup' is taken over rectangles in $\mathbf{R}^{n}$ congruent with $[0,6 \delta]^{n-1} \times$ $[0,6]$, of arbitrary direction and centered at $x$. Repeating carefully the proof of Theorem 2, one can obtain the sharper estimate

$$
\omega\left(\left\{x: \mathscr{M}_{\delta}\left(\chi_{A}\right)(x)>\sigma\right\}\right) \leq C_{n} \sigma^{-(n+1) / 2} \delta^{-(n-1) / 2} \mathscr{M}_{\delta}^{6} \omega(A)
$$

for all $A \subset B(0,1)$.

\section{ACKNOWLEDGMENT}

I would like to thank my adviser, F. Soria, for his help and friendship.

\section{REFERENCES}

[B] J. Bourgain, Besicovitch type maximal functions and applications to Fourier analysis, Geometric Funct. Anal. 1 (1991).

[CHS] A. Carbery, E. Hernández, and F. Soria, Proceedings ICM-90 Satellite Conference on Harmonic Analysis, Sendai (S. Igari, ed), Springer-Verlag, Tokyo, 1991, pp. 41-50.

[CDR] M. Christ, J. Duoandikoetxea, and J. L. Rubio de Francia, Maximal operator related to the Radon transform and the Calderon-Zygmund method of rotations, Duke Math. J. 53 (1986), 189-209. 
[C] A. Córdoba, The Kakeya maximal function and the spherical summation multipliers, Amer. J. Math. 99 (1977), 1-22.

[MS] D. Müller and F. Soria, A double-weight $L^{2}$ inequality for the Kakeya maximal function, preprint.

Departamento de Matemáticas, Facultad de Ciencias, Universidad Autónoma de MADRID, 28049 MADRID, SPAIN

E-mail address: vargas@vm1.uam.es 